\title{
Expression of sialic acids and other nonulosonic acids in Leptospira
}

\author{
Jessica N Ricaldi ${ }^{1,3}$, Michael A Matthias ${ }^{1}$, Joseph M Vinetz ${ }^{1}$ and Amanda L Lewis ${ }^{2,4^{*}}$
}

\begin{abstract}
Background: Sialic acids are negatively charged nine carbon backbone sugars expressed on mammalian cell surfaces. Sialic acids are part of a larger family of nonulosonic acid (NulO) molecules that includes pseudaminic and legionaminic acids. Microbial expression of sialic acids and other nonulosonic acids has been shown to contribute to host-microbe interactions in a variety of contexts, including participation in colonization, immune subversion, and behaviors such as biofilm formation, autoagglutination and motility. Previous research has suggested that some spirochetes may also express these molecules.
\end{abstract}

Results: Here we use a combination of molecular tools to investigate the presence of NulO biosynthetic gene clusters among clinical and saprophytic isolates of the genus Leptospira. Polymerase chain reaction and Southern blotting suggested that a variety of leptospires encoded NulO biosynthetic pathways. High performance liquid chromatography and mass spectrometry analyses provided biochemical evidence that di-N-acetylated NulO molecules are expressed at relatively high levels by L. interrogans serovar Lai strain 55601, and at lower levels by L. alexanderi serovar Manhao and L. fainei serovar Hurstbridge. Endogenous expression of $\mathrm{N}$-acetylneuraminic acid (Neu5Ac, the most common sialic acid) was documented in L. interrogans serovar Copenhageni strain L1-130. Neu5Ac biosynthesis is also supported by a unique gene fusion event resulting in an enzyme with an N-terminal $\mathrm{N}$-acetylneuraminic acid synthase domain and a C-terminal phosphatase domain. This gene fusion suggests that L. interrogans uses a Neu5Ac biosynthetic pathway more similar to animals than to other bacteria. Analysis of the composition and phylogeny of putative NulO biosynthetic gene clusters in L. interrogans serovar Lai and serovar Copenhageni revealed that both strains have complete biosynthetic pathways for legionamimic acid synthesis, a molecule with the same stereochemistry as sialic acid. Lectin-based affinity purification of NulO-modified molecules, followed by mass spectrometric identification suggests post-translational modification of surface lipoproteins, including Loa22.

Conclusions: Leptospira species encode NulO biosynthetic pathways and synthesize multiple NulO molecules including sialic acid. Additional studies are needed to clarify the exact context and functional significance of NulO expression. These findings have implications for immune evasion during systemic leptospirosis.

Keywords: Sialic acid, Nonulosonic acid, Leptospira

\footnotetext{
* Correspondence: allewis@wustl.edu

${ }^{2}$ Department of Pediatrics, University of California San Diego, School of

Medicine, La Jolla, CA 92093, USA

${ }^{4}$ Present address: Department of Molecular Microbiology, Washington

University School of Medicine, St. Louis, MO 63110, USA

Full list of author information is available at the end of the article
} 


\section{Background}

Leptospirosis, the most common zoonotic illness affecting humans, is caused by spirochetes of the genus Leptospira [1,2]. Some Leptospira species live exclusively in water or soil, while others cycle between environmental and mammalian reservoirs. Leptospira can colonize/ infect renal tubules of a wide variety of wild and domesticated mammals. Human disease follows exposure to water or soil contaminated with urine of infected animals. Leptospirosis can be asymptomatic, or manifest as a mild flu-like illness. In another subset of individuals (5-10 \% of patients) Leptospira can produce more serious systemic infections resulting in pulmonary hemorrhage, jaundice, renal failure, refractory shock, myocarditis, and/or aseptic meningitis.

Despite its medical importance, few virulence determinants of pathogenic Leptospira have been characterized in any detail. Investigation of the organism is hampered by its fastidiousness, slow growth in culture and the lack of available genetic tools. To date, only Omp-A like lipoprotein Loa22 has been demonstrated to be necessary for virulence, appearing to be cytotoxic and capable of inducing apoptosis. [3-5] LipL32, a major outer membrane protein of pathogenic Leptospira, is expressed in vivo and, although it has been shown to bind to host extra-cellular membrane, LipL32 does not seem to be required for acute or chronic infection in vivo in animal models. [6,7] Other potential virulence leptospiral factors include LigA and LigB that contain immunoglobulin-like repeats associated with adhesion to host cells in other gram-negative bacteria. Other proteins shown to have laminin binding activity in-vitro include LenA/LfhA/Lsf24 and related proteins LenBCDEF. LenA seems to also bind factor $\mathrm{H}$ of complement, so it might have more than one role in virulence. $[8,9]$. Leptospiral LPS, although not characterized in detail, has some unique characteristics which could explain why it is poorly recognized by the TLR4- MD2 complex. This diminished recognition could contribute to leptospiral survival in the bloodstream and dissemination. Other potential virulence factors for which more evidence remains to be published include mediators of motility and chemotaxis, including chemotaxis towards hemoglobin [10].

Sialic acids are a diverse family of acidic nine-carbon backbone (nonulosonic) monosaccharides found in abundance on the surfaces of mammalian cells and are sometimes expressed by microbial pathogens. The most common sialic acid in nature is $\mathrm{N}$-acetylneuraminic acid (Neu5Ac). Expression of Neu5Ac by pathogenic bacteria has been linked mechanistically to complement and neutrophil evasion in disseminated infections with Streptococcus and Neisseria and with the induction of autoimmune neuropathy following infection with Campylobacter. Sialic acids are part of an even wider family of di-N-acetylated nonulosonic acid (NulO) sugars, which also includes pseudaminic and legionaminic acids. Legionaminic acid was first described as part of the Legionella lipopolysaccharide O-antigen [11], which is thought to have roles in environmental and host associations [12]. Legionaminic and pseudaminic acids are also found as post-translational modifications of flagellin, best studied in Campylobacter and Helicobacter [13,14]. Even further, recent data suggest that in Helicobacter proteins other than flagellins may also undergo glycosylation [15]. Our recent genomic and phylogenetic analyses indicated the presence of NulO biosynthetic gene clusters in the available genomes of L. interrogans [16]. In this study, we sought to investigate the presence of $\mathrm{NulO}$ biosynthetic gene clusters in other Leptospira species and to determine whether these genes produced functional biosynthetic pathways. Here we define the presence of putative nonulosonic acid biosynthetic gene clusters in a variety of Leptospira species. Further biochemical investigations show that some Leptospira are capable of endogenous synthesis of nonulosonic acids, including sialic acids.

\section{Results and discussion}

Nonulosonic acid biosynthetic gene clusters are present among pathogenic and some intermediately pathogenic Leptospira species

The genome sequences of $L$. interrogans serovar Copenhageni strain L1-130 and L. interrogans serovar Lai strain 55601 contain genes predicted to synthesize sialic acids or related molecules (Figure 1A). Using PCR and Southern blotting, we evaluated the presence of this gene cluster in other isolates of Leptospira, including pathogenic, saprophytic, and intermediate strains. Polymerase chain reactions using primers designed from the genome strains amplified genes in the pathogenic strains L. interrogans serovar Copenhageni and Lai but not in the saprophyte L. biflexa (Figure 1B). Interestingly, one of the intermediate strains, $L$. licerasiae, gave a negative result, while the other, $L$. fainei, gave a faint positive. Control reactions using primers designed from $16 \mathrm{~S}$ rRNA gene showed amplification in all the samples, verifying DNA integrity. A probe based on the neuA2 gene of $L$. interrogans was used for southern blotting of genomic DNA from a number of Leptospira reference strains and isolates. These experiments confirm and extend the PCR data. Of particular interest is a pair of wild rodent isolates of Leptospira in lanes 6 and 7 (MMD4847 identifies as L. licerasiae and MMD3731 identified as $L$. interrogans serovar Copenhageni). Whereas the intermediately pathogenic $L$. licerasiae strain did not give a positive result, the pathogenic serovar Copenhageni isolate gave a strong positive band. Also, the intermediate strain $L$. fainei gave a positive result in southern blotting, further confirming the faint 


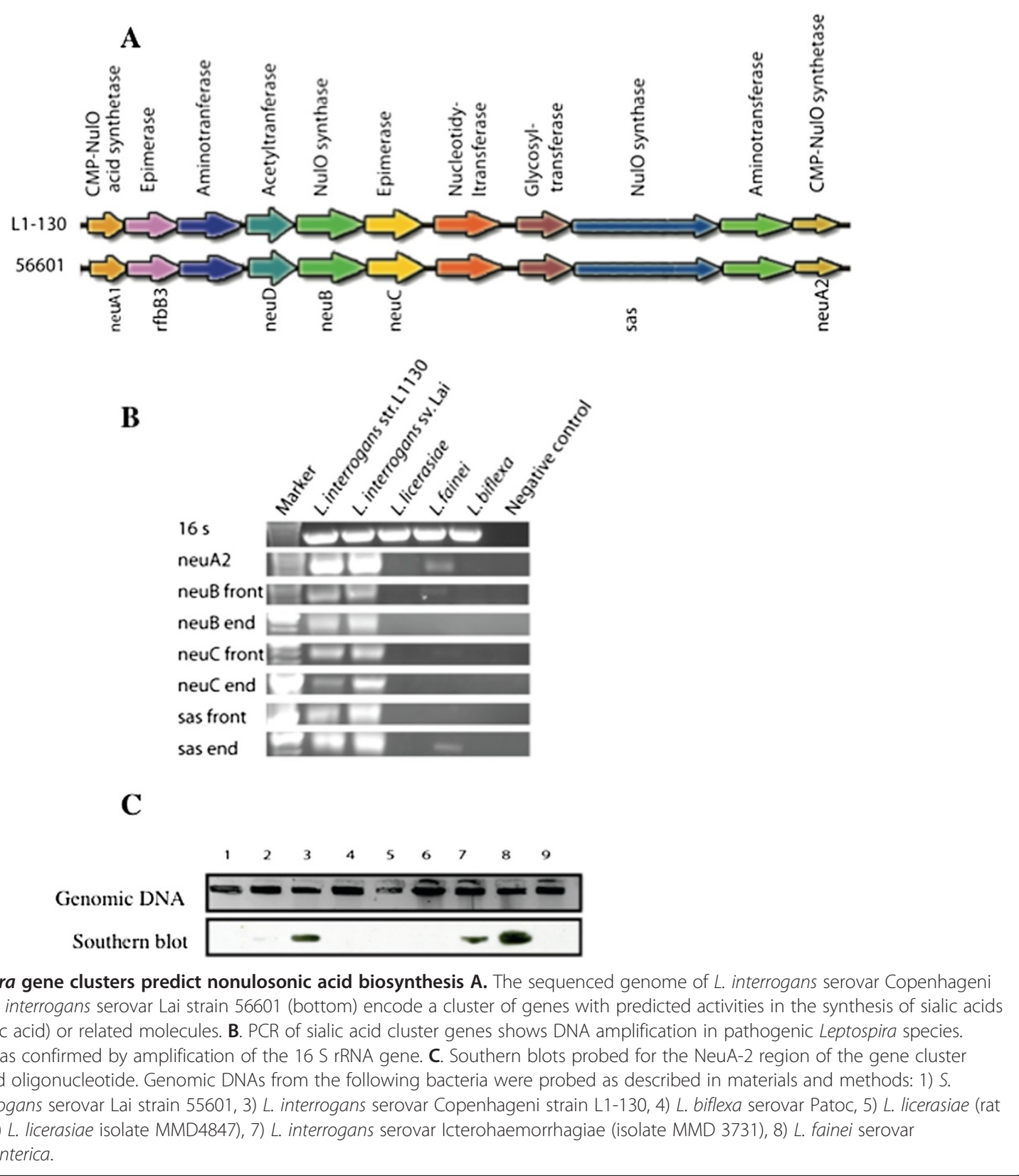

positive result observed by PCR for this strain. Since low sequence identity between primers or probes and the target sequences from less closely related species could produce a negative result in these experiments, other more functional assays were utilized next.

\section{DMB-derivatization and HPLC-MS analysis reveals multiple varieties of nonulosonic acids expressed by Leptospira}

Strains were evaluated biochemically to determine whether nonulosonic acid biosynthetic pathways were functional in different species and strains of Leptospira. Bacteria were hydrolyzed with mild acetic acid to release nonulosonic acid species, and low molecular weight fractions were fluorescently derivatized with 1,2-diamino4,5-methylene dioxybenzene (DMB), a molecule that specifically reacts with alpha keto acids, including NulOs. DMB-derivatized reaction products were separated by high performance liquid chromatography (HPLC) with a tandem electrospray ionization mass spectrometer. As expected by the Gram-negative-like structure of Leptospira, all samples displayed an earlyeluting HPLC peak corresponding to the retention time and mass of 2-keto-3-deoxy-D-manno-octulosonic acid (Kdo). Kdo is an 8 -carbon $\alpha$-keto acid present in the core region of lipopolysaccharide in most Gram-negative 


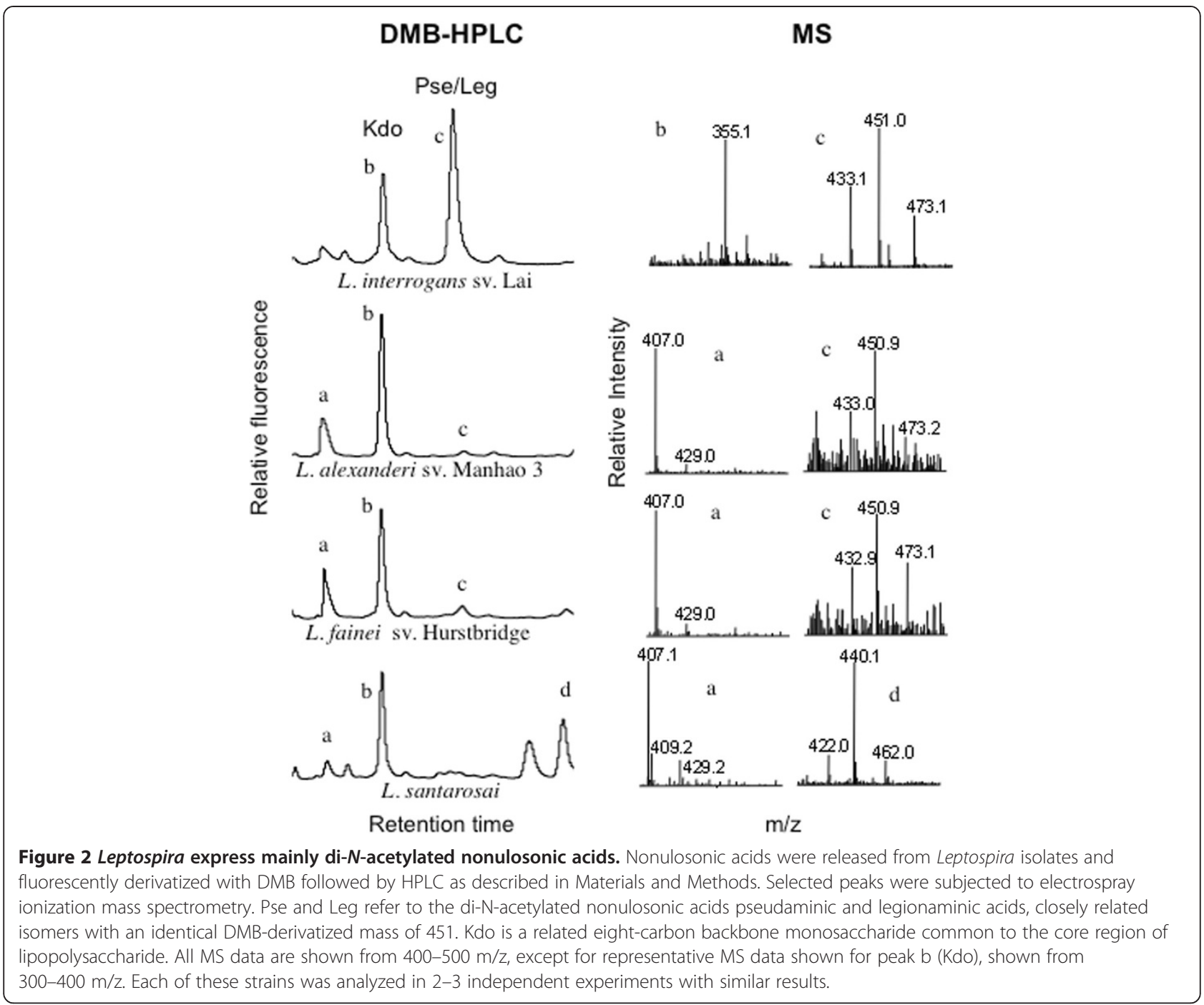

bacteria. It serves as an internal positive control in these assays (Figure 2 peak b, m/z 355) and allowed comparison between different HPLC runs. Masses of some DMB-derivatized peaks did not readily correspond to masses of known varieties of nonulosonic acids (for example Figure 2 peak a, 407 and peak d, 440). It is not known whether these masses represent nonulosonic acids. In contrast, a consistent $\mathrm{m} / \mathrm{z}$ of 433 (peak c) indicates the presence of di-N-acetylated nonulosonic acids and was found in pathogenic L. interrogans serovar Lai and $L$. alexanderi, and intermediate strain L. fainei. In all cases, the DMB-derivatized di-N-acetylated masses were accompanied with characteristic masses corresponding to the hydrated and hydrated sodium salt $(\mathrm{m} / \mathrm{z}$ 451 and 473 respectively). These biochemical data show that pathogenic and intermediately pathogenic strains of Leptospira are capable of expressing di-N-acetylated nonulosonic acids. However, in contrast, the pathogenic strain L. santarosai was not found to synthesize identifiable nonulosonic acid species at detectable levels (Figure 2). We also performed analyses on L. biflexa serovar Patoc. In this case, we observed the presence of Kdo by HPLC and mass spectrometry, but identifiable $\mathrm{NulO}$ molecules were not present at detectable levels (not shown).

Interestingly, HPLC analysis of the two different genome strains of L. interrogans (serovar Copenhageni strain L1-130 and serovar Lai strain 56601) gave distinct results. While L. interrogans serovar Lai expresses di-Nacetylated nonulosonic acid (Figure 2, m/z 433), strain L1-130 (serovar Copenhagenii) exhibited a peak with mass and retention time consistent with Neu5Ac $(\mathrm{m} / \mathrm{z}$ 408, hydrated 426, and hydrated sodium salt 448) (Figure 3A-B). Additional $\mathrm{MS}^{2}$ analysis consistently reduced this trio of masses almost exclusively to the parent mass of 408 (Figure 3B), as expected based on the behavior of standard Neu5Ac derivatized in parallel (Figure 3C). Since the common animal sialic acids 


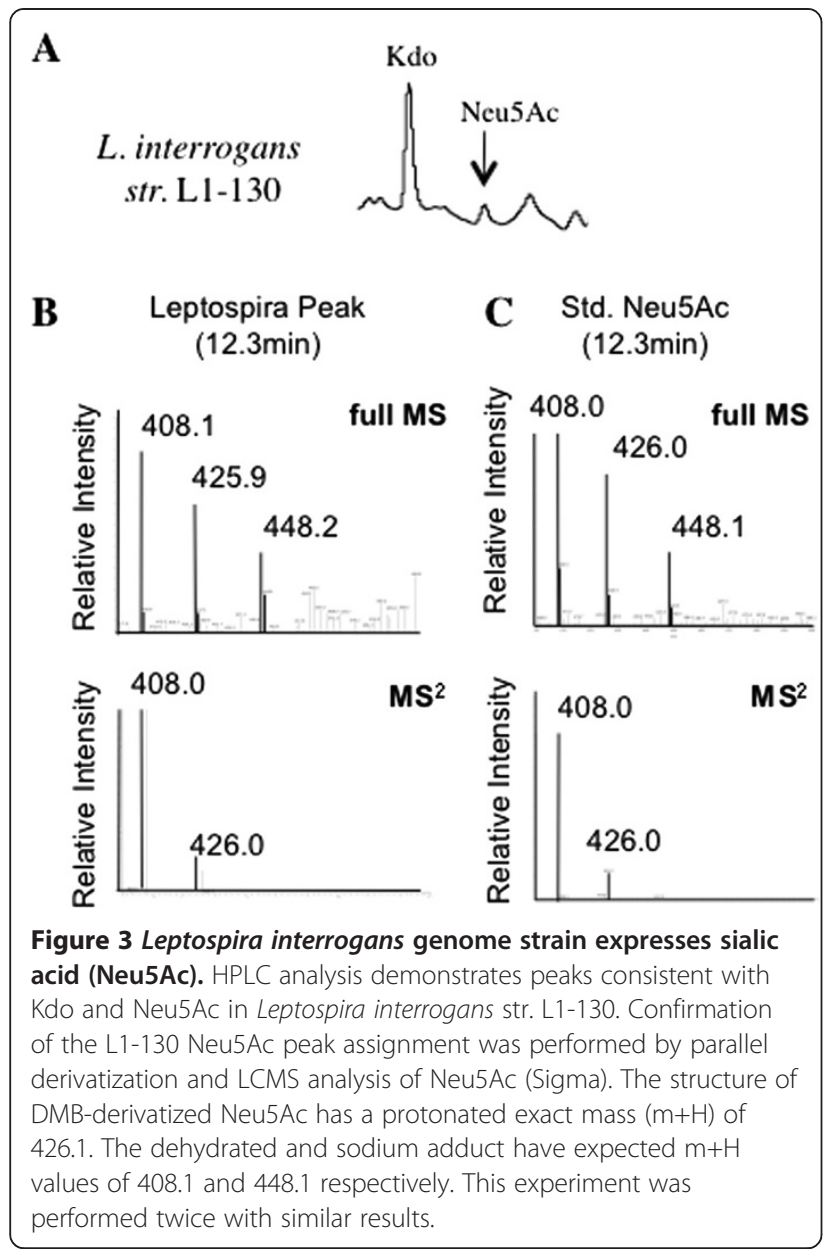

Neu5Ac and Neu5Gc were found in the standard culture media used for Leptospira (EMJH, Figure 4A), experiments were designed to exclude the possibility that $L$. interrogans strain L1-130 may incorporate exogenous sialic acid from the culture media. Unfortunately, the lack of a readily available genetic system for Leptospira rules out gene deletion as an approach to demonstrate endogenous synthesis. However, leptospires grown in defined serum-free media without sialic acids (as confirmed by HPLC) still produced a Neu5Ac peak, confirming that $L$. interrogans strain L1-130 synthesizes Neu5Ac and this sugar is not acquired from growth media (Figure 4B).

\section{Composition and phylogenetic analysis of NulO biosynthetic gene clusters and enzymes}

Next we performed analysis of the composition and phylogeny of the putative NulO biosynthetic gene clusters and the enzymes they encode in L. interrogans serovars Lai (strain 56601) and Copenhageni (strain L1-130). Consistent with the biochemical analysis of $L$. interrogans, genomic analysis of the $\mathrm{NulO}$ gene cluster reveals that the organism encodes a complete pathway for di-Nacetylated nonulosonic acid biosynthesis (see Table 1 in comparison with Figure 5). There are multiple distinct open reading frames encoding synthesis of aminotransferases, NulO synthases, and CMP-NulO synthetases (see Table 1 and Figure 5), suggesting that $L$. interrogans may express multiple nonulosonic acid species, a conclusion supported by our biochemical investigations (Figure 2 and Figure 3).

Phylogenetic comparisons were performed to provide additional insights into the potential functions of Leptospira nonulosonic acid biosynthesis enzymes. We included in the phylogenetic analysis the wellcharacterized enzymes of Campylobacter jejuni that participate in parallel pathways of legionamimic, pseudaminic, and neuraminic acid synthesis [14,17-21]. A schematic of these biosynthetic pathways is shown in Figure 5, noting the structural differences between neuraminic (sialic), legionamimic, and pseudaminic acids. These different NulOs are used by $C$. jejuni to modify a variety of different surface structures including the Oantigen of lipooligosaccharides, flagellin, and other surface proteins. To add further resolution to our phylogenetic analysis, we also included NulO biosynthetic enzymes from two Photobacterium profundum genome strains (3TCK and SS9), previously demonstrated to synthesize legionamimic and pseudaminic acids respectively [16]. In addition, homologous enzymes from other Leptospira genomes (L. noguchii str. 2006001870, L.

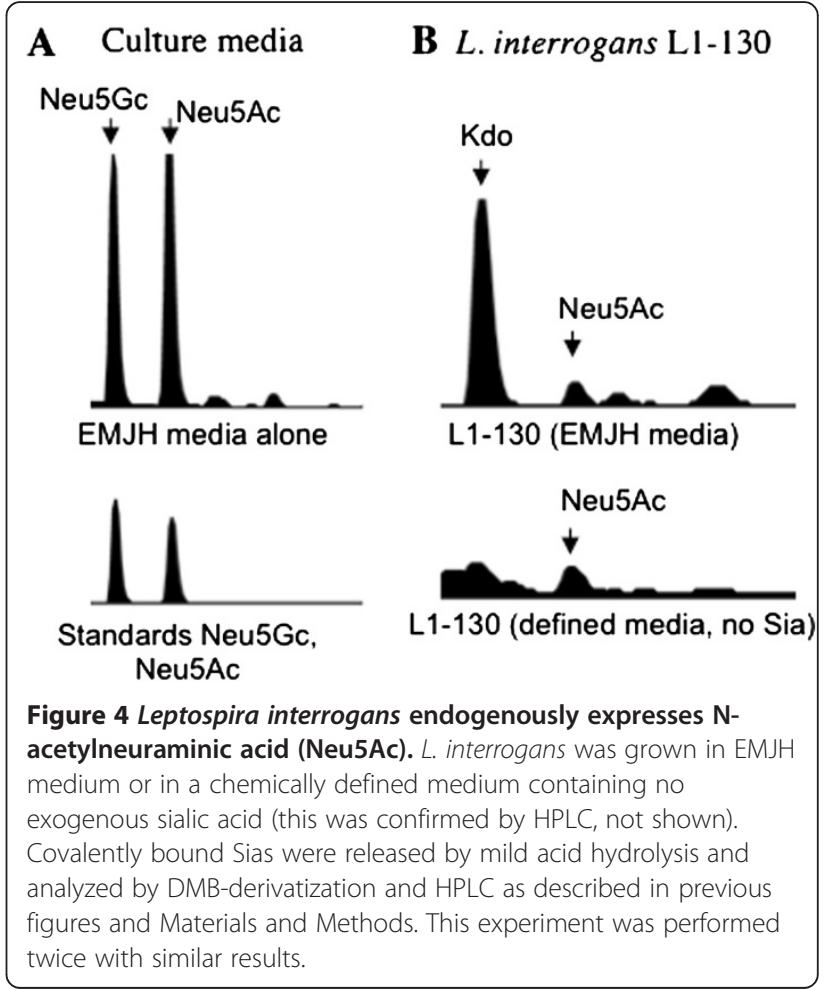


Table $1 L$. interrogans encodes a complete pathway for legionaminic acid synthesis

\begin{tabular}{|c|c|c|c|c|}
\hline $\begin{array}{l}\text { Campylobacter enzymes } \\
\text { for legionaminic } \\
\text { acid biosynthesis } \\
{[14,17-21]}\end{array}$ & $\begin{array}{l}\text { C. jejuni } \\
\text { Pathway number } \\
\text { (Figure 5) }\end{array}$ & $\begin{array}{l}\text { L. interrogans } \\
\text { L1-130 \& 56601 } \\
\text { NCBI accession } \\
\text { numbers }\end{array}$ & $\begin{array}{l}\text { Predicted } \\
\text { L. interrogans } \\
\text { Pathway number } \\
\text { (Figure 5) }\end{array}$ & Predicted enzymatic Function \\
\hline \multirow[t]{2}{*}{ PmtE (cj1329) } & \multirow[t]{2}{*}{1} & YP_002106 & \multirow[t]{2}{*}{1} & \multirow[t]{2}{*}{ Glc-1-P guanyltransferase } \\
\hline & & NP_711792 & & \\
\hline \multirow[t]{2}{*}{$\mathrm{GlmU}$} & \multirow[t]{2}{*}{2} & YP_000413 & \multirow[t]{2}{*}{2} & (housekeeping) \\
\hline & & NP_714003 & & $\mathrm{N}$-acetyltransferase \\
\hline \multirow[t]{2}{*}{ LegB (cj 1319) } & \multirow[t]{2}{*}{3} & YP_002111 & \multirow[t]{2}{*}{3} & \multirow[t]{2}{*}{ 4,6-dehydratase } \\
\hline & & NP_7111787 & & \\
\hline \multirow[t]{4}{*}{ LegC (cj1320) } & \multirow[t]{4}{*}{4} & YP_002110 & \multirow[t]{2}{*}{4} & \multirow{2}{*}{$\begin{array}{l}\text { Aminotransferase in } \\
\text { legionaminic acid synthesis } \\
\text { (Figure 6A) }\end{array}$} \\
\hline & & NP_7111788 & & \\
\hline & & YP_002103 & \multirow[t]{2}{*}{4,13, or ? } & \multirow[t]{2}{*}{ Aminotransferase } \\
\hline & & NP_711795 & & \\
\hline \multirow[t]{2}{*}{ LegH (cj1298) } & \multirow[t]{2}{*}{5} & YP_002109 & \multirow[t]{2}{*}{5} & \multirow[t]{2}{*}{$\mathrm{N}$-acetyltransferase } \\
\hline & & NP_711789 & & \\
\hline \multirow[t]{2}{*}{ LegG (cj1328) } & \multirow[t]{2}{*}{6} & YP_002107 & \multirow[t]{2}{*}{6} & \multirow{2}{*}{$\begin{array}{l}\text { 2-epimerase/NDP sugar } \\
\text { hydrolase in legionamimic } \\
\text { acid synthesis }\end{array}$} \\
\hline & & NP_711791 & & \\
\hline \multirow[t]{4}{*}{ Legl (cj1327) } & \multirow[t]{4}{*}{7} & YP_002108 & \multirow[t]{2}{*}{7} & \multirow{2}{*}{$\begin{array}{l}\text { Legionaminic acid synthase } \\
\text { (Figure 6B) }\end{array}$} \\
\hline & & NP_711790 & & \\
\hline & & YP_002104 & \multirow[t]{2}{*}{10} & \multirow{2}{*}{$\begin{array}{l}\text { Legionaminic or neuraminic } \\
\text { acid synthase (Figures } 6 B \& 7 \text { ) }\end{array}$} \\
\hline & & NP_711794 & & \\
\hline \multirow[t]{4}{*}{ LegF (cj1331) } & \multirow[t]{4}{*}{8} & YP_002102 & \multirow[t]{2}{*}{8 or 11} & \multirow{4}{*}{$\begin{array}{l}\text { CMP-Legionaminic acid or } \\
\text { neuraminic acid synthetases } \\
\text { (Figure 6C) }\end{array}$} \\
\hline & & NP_711796 & & \\
\hline & & YP_002112 & 8 or 11 & \\
\hline & & NP_711786 & & \\
\hline
\end{tabular}

biflexa serovar Patoc, L. santarosai str. 2000030832, L. borgpetersenii serovar Hardjo-bovis str. L550) were included in the phylogenetic analysis to better place the L. interrogans NulO enzymes into context with other putative leptospiral NulO biosynthetic enzymes.

The phylogenetic analysis of L. interrogans NulO biosynthetic enzymes demonstrates that a subset of these enzymes is more closely related to the $C$. jejuni legionaminic acid biosynthetic enzymes and more distantly related to the pseudaminic acid biosynthetic enzymes (Figure 6). Specifically, the aminotransferases YP_002110 and NP_711788 and the NulO synthetases YP_002108 and NP_711790 in L. interrogans serovars Copenhageni and Lai respectively, are more closely related to legionaminic acid synthesis enzymes and more distantly related to $C$. jejuni and P. profundum pseudaminic acid synthesis enzymes (Figure 6A-B, note green and pink shading indicates legionaminic acid pseudaminic acid pathways respectively). A similar relationship was found for the predicted epimerase/NDP-sugar hydrolases YP_002107 and NP_711791(not shown). Moreover, we find that both homologs of the putative CMP-NulO synthetases in L. interrogans (YP_002102 and YP_002112 in L1-130 and NP_711786 and NP_711796 in 56601) are more closely related to legionaminic acid and neuraminic acid synthetases than to CMP-pseudaminic acid synthetases (Figure 6C). Note in this figure that CMP-Kdo synthases were included to provide contrast and distinguish between enzymes that likely participate in CMP activation of eight carbon sugars (i.e. Kdo) and nine carbon sugars (i.e. NulOs). The sequences retrieved by BLAST of these Leptospira genomes, together with their phylogeny, suggest that a number of leptospires do not encode homologs of CMP-NulO synthetases. In contrast, some leptospires encode putative NulO biosynthesis enzymes that are more closely related to the $C$. jejuni and $P$. profundum pseudaminic acid biosynthesis enzymes and more distantly related to the legionaminic acid enzymes (e.g. L. noguchii Figure 6A-B).

In contrast to bacterial $\mathrm{NulO}$ biosynthetic pathways that synthesize Neu5Ac from ManNAc (N-acetyl mannosamine), the mammalian pathway relies on a $\mathrm{NulO}$ synthase with unique specificity for 6-phosphatemodified ManNAc, to generate 9-phosphate-modified 


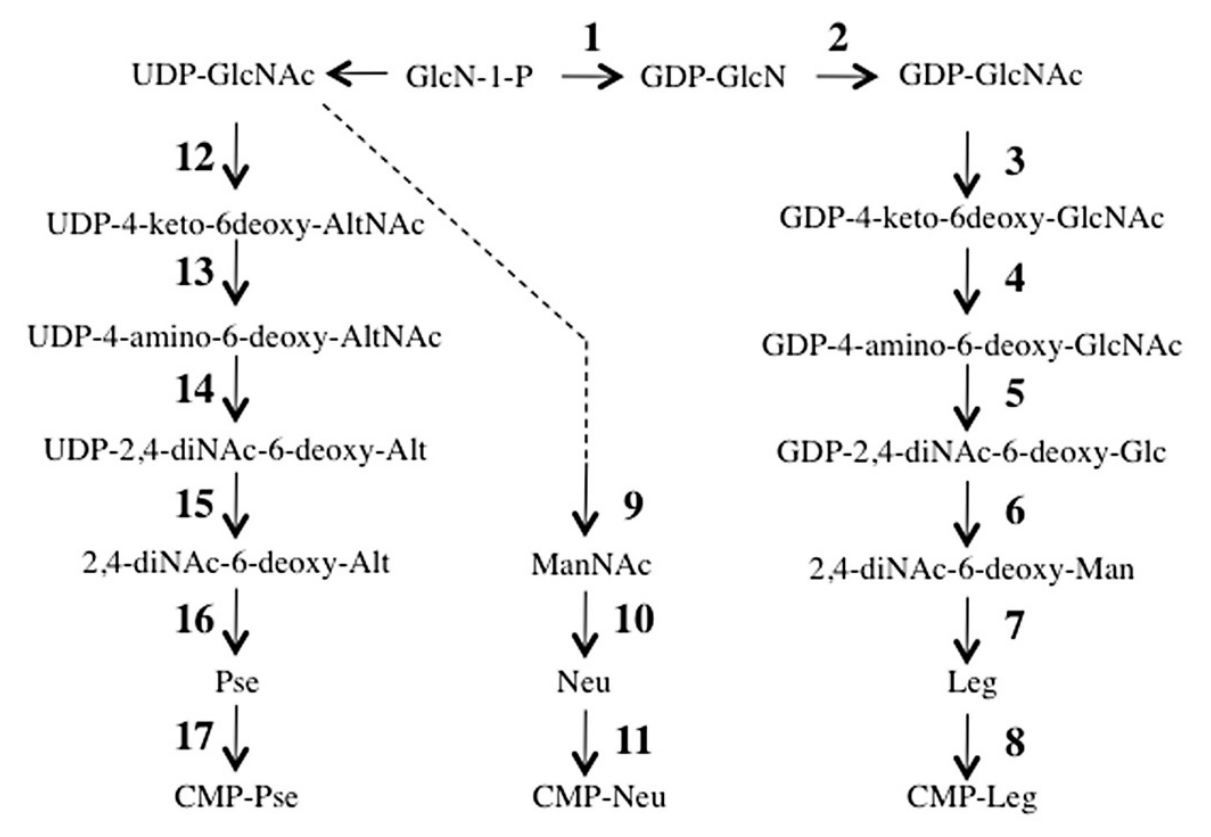

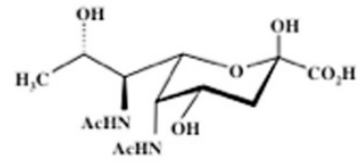

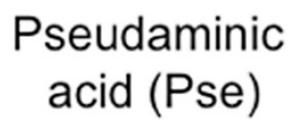

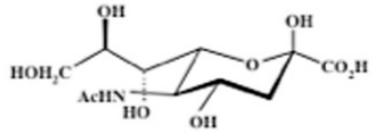

Neuraminic acid (Neu)

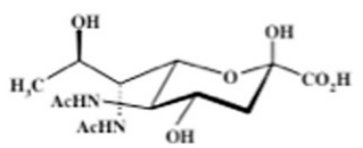

\section{Legionaminic acid (Leg)}

Figure 5 Schematic of pseudaminic, legionamimic, and neuraminic acid biosynthetic pathways. Studies of nonulosonic acid biosynthesis at the enzymatic level have been carried out with greatest resolution using C. jejuni and H. pylori as model systems $[14,17-21,35]$. Note that parallel arrows in this diagram indicate distinct, yet homologous enzymes. This schematic is based largely on the work of Schoenhofen et. al. Please refer to $[14,18]$ and references within for more detailed descriptions of the enzymes and intermediates of these pathways.

Neu5Ac [22]. A set of adapter enzymes precede (kinase) and follow (phosphatase) the NulO synthase in the animal pathway (see Figure 7). In some cases, 'adapter' enzymes have become fused into the same open reading frame with one of the other nonulosonic acid biosynthesis genes. One example is the mammalian UDPGlcNAc-2-epimerase, which is fused to a kinase that phosphorylates ManNAc to generate the substrate for the next step of the pathway, ManNAc-6-P. Interestingly, when performing analyses of $L$. interrogans $\mathrm{NulO}$ biosynthetic pathway, we noted that one of the NulO synthases encoded by L. interrogans (YP_002104 in serovar Copenhageni and NP_711794 in serovar Lai) has a unique C-terminal domain that is homologous to endonucleases that cleave phosphodiester bonds. By inference, we suggest that the route for $\mathrm{N}$-acetylneuraminic acid biosynthesis in $L$. interrogans may be very similar to the animal pathway, condensing phosphoenolpyruvate with a phosphorylated 6-carbon intermediate to generate a phosphorylated 9-carbon sugar, followed by dephosphorylation catalyzed by the fused C-terminal phosphatase domain (Figure 7). This enzyme is distantly related to other NulO synthases and did not cluster with animal neuraminic acid synthases when these enzymes were included in the analysis (not shown), suggesting that this biosynthetic route may be ancestral. This conclusion is supported by previous evolutionary analyses of NulO pathways [16].

\section{Nonulosonic acids are elaborated on Leptospira surface lipoproteins}

Finally, efforts were made to identify the type of molecule (s) modified with sialic acids in L. interrogans strain L1-130. Immobilized sialic acid-binding lectins from Sambucus nigra agglutinin (SNA) and Maackia amurensis lectin (MAL), which recognize sialic acids in $\alpha 2-6$ and $\alpha 2-3-$ linked sialic acids respectively, were used to affinity purify sialic acid-modified molecules in lysates of the L1-130 strain. Wheat germ agglutinin (WGA) also recognizes sialic acids, but is less specific, and also recognizes $\mathrm{N}$ - 


\section{A Aminotransferase homologs}

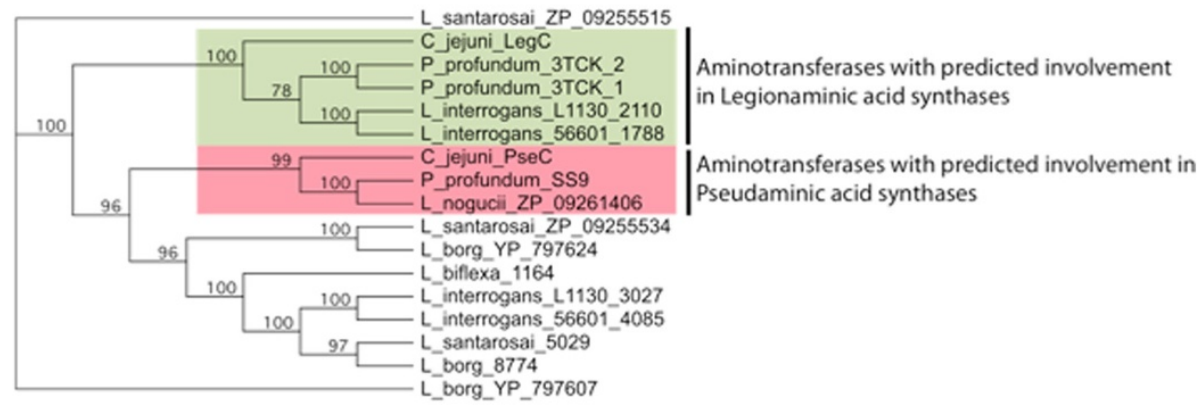

\section{B NulO synthase homologs}

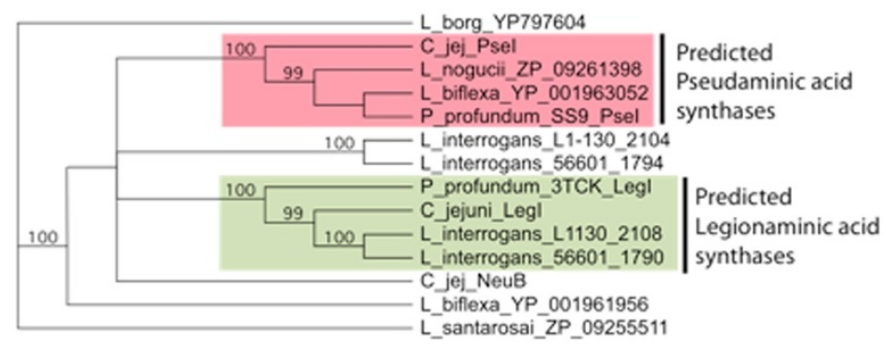

\section{CMP-NulO synthetase homologs}

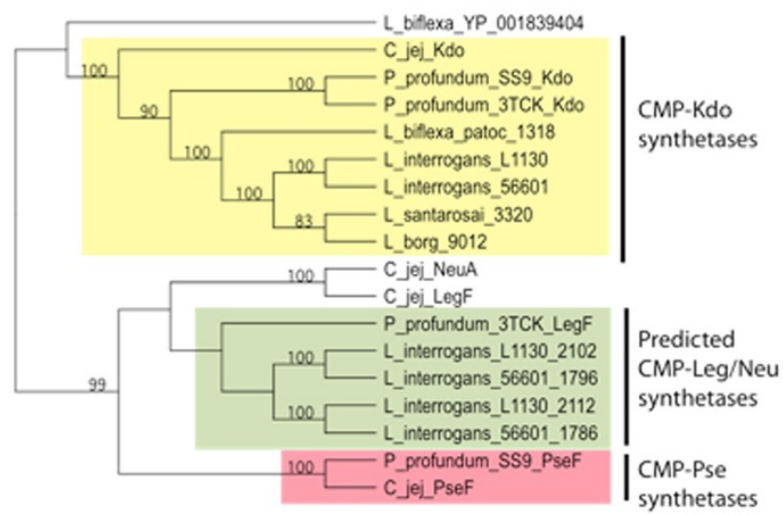

Figure 6 Phylogenetic analysis of $L$. interrogans NulO biosynthetic enzymes. Amino acid sequence alignments of "aminotransferase," "NulO synthase," and "CMP-NulO synthetase," enzymes were performed using Clustal W and phylogenetic trees were built using the Neighbor-Joining method. Campylobacter jejuni enzymes with characterized functions in bacterial neuraminic, legionaminic, and pseudaminic acid biosynthesis $[14,17-21]$ were compared to L. interrogans amino acid sequences encoded in the NulO biosynthetic gene cluster. Homologs of these enzymes from P. profundum strains 3TCK and SS9 were also included as they are know to synthesize legionamimic acid pseudaminic acids respectively [16]. Homologous enzymes from other selected Leptospira genomes (L. noguchii str. 2006001870, L. biflexa serovar Patoc, L. santarosai str. 2000030832, L. borgpetersenii serovar Hardjo-bovis L550) were also included in the phylogenetic analysis.

acetylglucosamine residues. As a control, buffers used in the solid phase assay were analyzed in parallel lanes of the gel, revealing that the faint bands present at $\sim 60 \mathrm{kDa}$ were part of the supplied buffers and not specific for sialylated $L$. interrogans molecules. Silver staining after SDS-Page gel electrophoresis of the eluted material from the affinity columns shows clear bands at $\sim 21 \mathrm{kDa}$ and $\sim 25 \mathrm{kDa}$ that are present at similar intensities in the MAL and SNA lanes
(Figure 8A). Other bands appear to be enriched by affinity purification using one or the other lectin. For example, a faint band at $\sim 43 \mathrm{kDa}$ is apparent in the material isolated by MAL, but not by SNA. Alternatively, bands at 15, 37, and $\sim 41 \mathrm{kDa}$ are much stronger in the SNA-purified sample. These finding suggests that $L$. interrogans may modify surface structures with both $\alpha 2-3$ - and $\alpha 2-6$-linked nonulosonic acids (Figure 8A). However, future studies should 

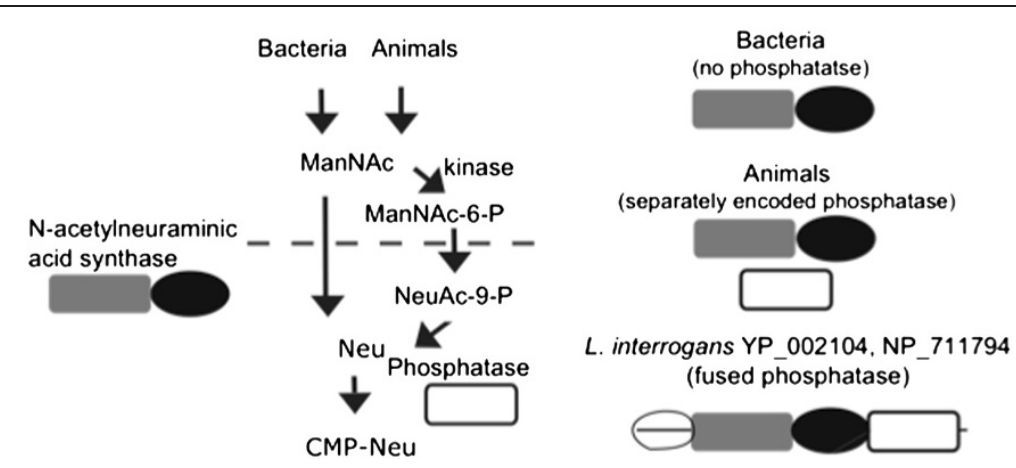

Figure 7 C-terminal phosphatase domain fused to putative $\mathrm{N}$-acetylneuraminic acid synthase suggests an animal-like Neu5Ac biosynthetic pathway in L. interrogans.

further investigate the molecule(s) modified by nonulosonic acids in leptospires, as well as their exact context and importance.

The affinity-purified material was subjected to DMBderivatization and HPLC analysis, which showed the Neu5Ac peak, but not the Kdo peak (data not shown), strongly suggesting that this material was free of LPScomponents. This does not rule-out that LPS may be modified with NulOs, just that LPS was not present in this affinity-purified preparation. We performed mass spectrometry to identify protein components in the affinitypurified material. Three proteins were identified by mass spectrometry (Figure 8B): Loa22, LipL32, and LipL41, all of which have been described in previous publications as surface-exposed lipid-linked outer membrane proteins of L. interrogans [23-27]. Indeed, Loa22 and LipL31 are among the most abundant proteins expressed on the Leptospira cell surface [28]. Loa22 was identified with the highest number of peptide matches. Loa22 is an outer membrane protein encoded within all Leptospira genomes sequenced to date. It has been observed to be upregulated in vivo [27] and it is one of very few leptospiral proteins so far that has been shown to be necessary for virulence [3]. Additional studies are needed to define the precise context of NulO expression on L. interrogans and understand its potential significance in virulence.

\section{Conclusions}

Based on a combination of experimentation and in silico genomic analysis, we have demonstrated the function of $\mathrm{NulO}$ biosynthetic gene clusters in pathogenic and intermediately pathogenic species of Leptospira, several of which are capable of synthesizing di- $\mathrm{N}$-acetylated $\mathrm{NulO}$ species, as well as the true sialic acid, $\mathrm{N}$-acetyneuraminic acid, a finding of considerable consequence for the leptospirosis field. This finding expands the number of important human pathogens that utilize endogenous biosynthetic pathways to elaborate surface structures containing sialic acids and related NulO molecules [16]. Sialic acids have proven roles in complement evasion, intracellular survival, and biofilm formation [29], and evidence is emerging that some human pathogens with Neu5Ac on their surfaces can engage sialic acid-binding receptors (Siglecs) on leukocyte cell surfaces, resulting in phagocytosis or

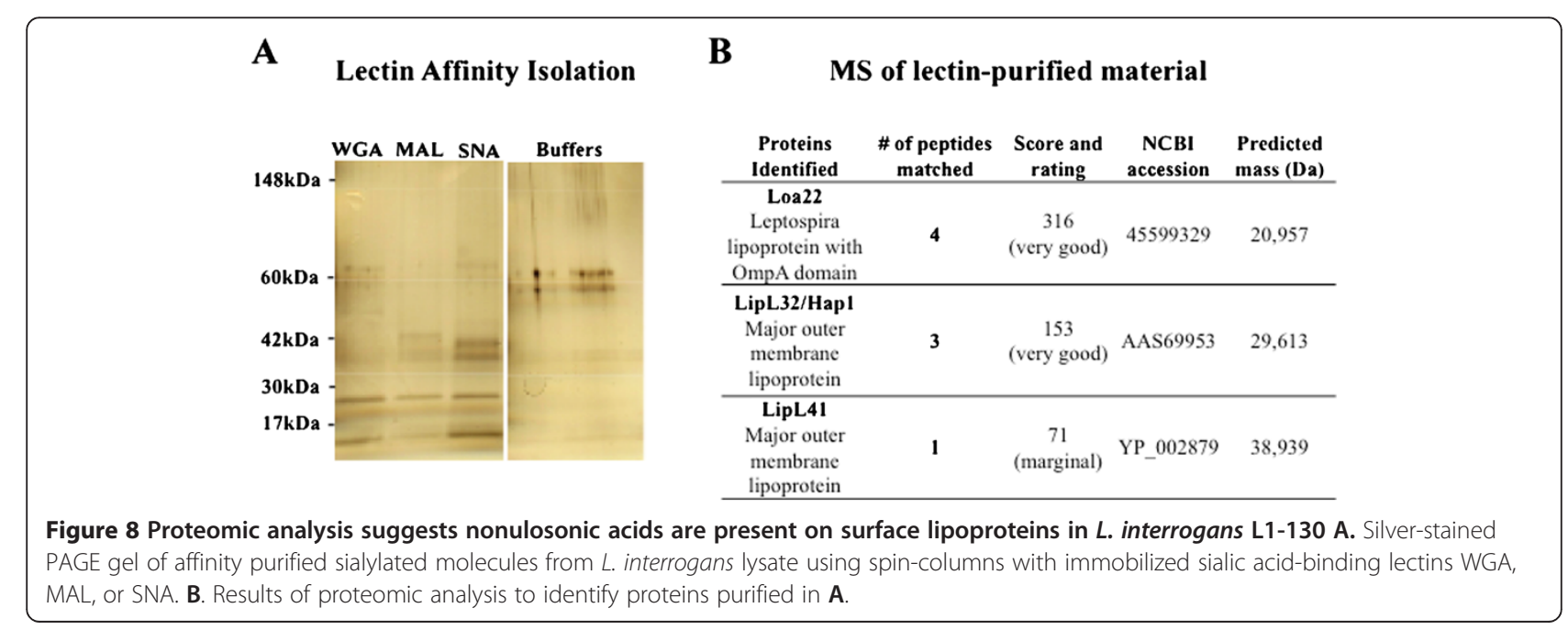


dampening of bactericidal activities [30-32]. The roles of other NulO molecules such as legionaminic and pseudaminic acids are less well defined, but these molecules have been shown to play roles in behaviors such as autoagglutination, motility, and host colonization [33-37]. Curiously, disease caused by L. interrogans includes bacteremia and meningitis as components of the clinical disease spectrum, similar to the well-characterized Neu5Ac-expressing human bacterial pathogens Group B Streptococcus, Neisseria meningitidis, E. coli $\mathrm{K} 1$, and Haemophilus influenzae. As genetic tools and small animal infection systems for study of Leptospira are further refined, analysis of the contribution of NulO biosynthesis to the virulence of this neglected disease can be further elucidated.

\section{Methods}

\section{Strains and culture conditions}

Intermediately pathogenic strains $L$. licerasiae serovar Varillal strains MMD3731, MMD4847 and CEH008 (isolated from rodents in Peru), L. fainei serovar Hurstbridge strain BUT $6^{\mathrm{T}}$ and the saprophyte $L$. biflexa serovar Patoc were used for these experiments. Pathogenic Leptospira used in this study included L. interrogans serovar Copenhageni strain L1-130, L. interrogans serovar Lai strain 55601, and L. interrogans serovar Icterohaemorrhagiae wild rodent isolate MMD 3731 that were passaged fewer than 5 times in vitro after re-isolation from hamster liver to maintain virulence. $L$. santarosai and $L$. alexanderi serovar Manhao were originally isolated from clinical cases of leptospirosis and now serve as reference laboratory strains. Generally, Leptospira were cultivated at $30^{\circ} \mathrm{C}$ in Ellinghausen-McCulloughJohnson-Harris (EMJH) medium (catalog \#279510, Becton Dickinson, Sparks, Maryland). Chemicallydefined, sialic acid-free medium, prepared as previously described and verified by HPLC to be sialic acid free, was used to cultivate Leptospira in experiments where the lack of exogenous sialic acids was a necessary condition [38].

\section{PCR of sialic acid cluster genes}

Primers based on the genome of L. interrogans L1130 were designed for the detection of genes in the sialic acid cluster as follows: sasfrontF $\left(5^{\prime}-\right.$ TCC GGA AAT GCG AAT GAT G-3'), sasfrontR (5' - CAC CGG GCA AAA GAC TAA CCT - 3'), sasendF (5' CGG ATA TAG CGG ACG ATG TAA - 3'), sasendR (5' - CGC CAA AAA GCC AAG GAA - 3'), neuA2F (5' - TGA AGC GGC AAA AAG AGC - 3'), neuA2R (5' - TGA AAT AAC ATG CCG ACA AAT A - 3'), neuCfrontF (5'- CGC TAC GGG AAT GCA TCT GTC TC - 3'), neuCfrontR (5'- CCC ATT CCC CCA ACC AAA AA - 3'), neuCendF (5'- GGC GAG
GAT CCT TCT AAT GTT TTT - 3') and neuCendR (5' - ACT CGC TCC GCC TTC ACC A - 3'). PCR reactions were prepared using $0.2 \mathrm{mM}$ of each primer in a $20 \mu \mathrm{L}$ reaction with DNA from the pathogens $L$. interrogans Lai, L. interrogans L1-130, the intermediates $L$. licerasiae and $L$. fainei and the saprophyte $L$. biflexa serovar Patoc. NeuA2 and neuBfront reactions used an annealing temperature of $52^{\circ} \mathrm{C}$. NeuCfront, neuCend, sasfront and sasend were run using an annealing temperature of $58^{\circ} \mathrm{C}$. A $16 \mathrm{~S}$ gene PCR reaction using previously published primers fLIP and rLIP1 was used as a control for integrity of DNA.

\section{NeuA2 southern blot}

Genomic DNA samples of Salmonella enterica, $L$. interrogans serovar Lai str. 56601, L. interrogans serovar Copenhageni str. L1-130, L. biflexa serovar Patoc, L. licerasiae strains CEH008 and MMD4847, L. interrogans serovar Icterohaemorrhagiae str.MMD3731 and L. fainei serovar Hurstbridge were prepared into plugs using $1 \%$ agarose and $0.5 \mathrm{x}$ TBE. These were subjected to depurination and denaturing conditions. DNA was then transferred to a positively charged membrane via overnight capillary transfer with $20 \mathrm{x}$ SSC. Finally the DNA was cross-linked to the membrane using short wave DNA for $15 \mathrm{~min} .10 \mathrm{~mL}$ of pre-hybridization solution (QuikHyb, Stratagene) were warmed to $40^{\circ} \mathrm{C}$ prior to hybridization. Hybridization was done overnight at $40-42^{\circ} \mathrm{C}$ using the same solution and adding $10 \mathrm{~mL}$ of DIG-labeled PCR product of primer neuA2F (5' - TGA AGC GGC AAA AAG AGC - 3') and neuA2R (5 '- TGA AAT AAC ATG CCG ACA AAT A - 3'). 2xSSC at room temperature and $1 \mathrm{x}$ SSC at $68^{\circ} \mathrm{C}$ were used for stringency washes. A chemiluminescent substrate and an alkaline phosphatase conjugated anti-DIG antibody were used to demonstrate binding of the probe.

\section{Mild acid hydrolysis and DMB-derivatization of nonulosonic acids}

Mild (2 N) acetic acid hydrolysis was performed to release surface nonulosonic acids from Leptospira. $4 \mathrm{~N}$ acetic acid was added to an equal volume of extensively washed and resuspended pellets followed by $3 \mathrm{~h}$ of incubation at $80^{\circ} \mathrm{C}$. The resulting soluble fraction was filtered to remove large molecular weight components and derivatized with DMB (1,2-diamino4,5-methylene dioxybenzene), a reagent that reacts with the $\alpha$-keto acid portion of nonulosonic acids. Final reaction conditions were $7 \mathrm{mM}$ DMB, $18 \mathrm{mM}$ sodium hydrosulfite, 1.4 $\mathrm{M}$ acetic acid, and $0.7 \mathrm{M} 2$ mercaptoethanol. Derivatization was carried out for 2 hours at $50^{\circ} \mathrm{C}$ in the dark. 


\section{High performance liquid chromatography and mass spectrometry}

DMB-NulO derivatives were resolved by HPLC using a reverse phase C18 column (Varian) eluted isocratically at a rate of $0.9 \mathrm{ml} / \mathrm{min}$ over 50 minutes using $85 \%$ MQwater, $7 \%$ methanol, $8 \%$ acetonitrile as previously described $[16,39,40]$. In some experiments, HPLC was performed without online mass spectrometry and detection of fluorescently labeled NulO sugars was achieved using an online fluorescence detection using excitation and emission wavelengths of $373 \mathrm{~nm}$ and $448 \mathrm{~nm}$ respectively. In other experiments HPLC was combined with online mass spectrometry using a Thermo-Finnigan model LCQ ion trap mass spectrometer system. When mass spectrometry was performed, the mobile phase also included $0.1 \%$ formic acid, and online UV detection of DMB-NulO molecules preceded mass spectrometric analysis. We note that similar HPLC-MS analyses have been described previously DMB-derivatized $\alpha$-keto acids [39-41].

\section{Phylogenetic analysis}

We performed BLAST searches (blastp) against the NCBI genome database using as seeds the sequences of 1) proteins encoded by Campylobacter jejuni pseudaminic, legionaminic, and neuraminic acid biosynthetic pathways or 2) enzymes encoded in the Leptospira interrogans NulO biosynthetic gene cluster (Figure 1A). NCBI accession numbers are provided in Table 1 and a schematic of the biosynthetic pathways is illustrated in Figure 5. Complete protein sequences of homologous amino acids were aligned using ClustalW in MacVector 11.1.1 software and alignments were checked manually. The Neighbor Joining (NJ) method was utilized for phylogenetic tree construction using MacVector 11.1.1 software, including 1000 Bootstrap replications to obtain confidence values for branches of the NJ trees.

\section{Solid-phase lectin binding}

Whole cell lysates were prepared using three cycles of freeze-thawing of PBS washed L. interrogans serovar Copenhageni strain L1-130. In order to probe the abundance and nature of the sialylated molecules on $L$. interrogans, these lysates were fractionated using a lectinbased solid phase assay (Q Proteome Sialic Acid kit, Qiagen) using three immobilized sialic acid binding lectins: wheat germ agglutinin (WGA), Sambucus nigra agglutinin (SNA), and Maackia amurensis lectin (MAL), according to manufacturer's instructions. Molecules captured by each of these lectins were eluted according to the manufacturers instructions. then analyzed by SDSPAGE followed by silver staining (SilverQuest Silver Staining Kit, Invitrogen).

\section{Mass spectrometry}

To determine whether $L$. interrogans uses nonulosonic acids for post-translational modification of proteins, pooled affinity-purified material from above mentioned experiment was subjected to denaturation, reduction, and alkylation, followed by trypsin digestion and MS/MS analysis using a nano-flow LC- tandem mass spectrometer. Peptide mass fingerprints identified in the affinitypurified material were used to identify L. interrogans proteins by searching against the NCBInr bacterial genome database.

\section{Competing interests}

The authors declare that they have no competing interests.

\section{Authors' contributions}

JNR, MAM, JMV and ALL conceived and designed experiments, JNR and ALL acquired data, JNR, MAM and ALL analyzed and interpreted data, JNR and ALL drafted manuscript, JNR, MAM, JMV, and ALL revised manuscript critically for important intellectual content. All authors read and approved the final manuscript.

\section{Acknowledgments}

We thank Ajit Varki and Victor Nizet for valuable advice and Sandra Diaz for technical assistance with HPLC-MS. The Scripps Research Institute's Center for Mass Spectrometry performed nano-flow MS/MS data and provided results of the NCBInr database search (http://masspec.scripps.edu/). This work was supported in part by U.S. Public Health Service grants from the National Institutes of Health 1D43TW007120 and 1RO1TW05860.

\section{Author details}

${ }^{1}$ Department of Medicine, Division of Infectious Diseases, University of California San Diego, School of Medicine, La Jolla, CA 92093, USA.

${ }^{2}$ Department of Pediatrics, University of California San Diego, School of Medicine, La Jolla, CA 92093, USA. ${ }^{3}$ Present address: Instituto de Medicina Tropical Alexander von Humboldt, Universidad Peruana Cayetano Heredia, Lima, Peru. ${ }^{4}$ Present address: Department of Molecular Microbiology, Washington University School of Medicine, St. Louis, MO 63110, USA.

Received: 6 February 2012 Accepted: 19 July 2012

Published: 1 August 2012

\section{References}

1. Bharti AR, Nally JE, Ricaldi JN, Matthias MA, Diaz MM, Lovett MA, Levett PN, Gilman RH, Willig MR, Gotuzzo E, et al: Leptospirosis: a zoonotic disease of global importance. Lancet Infect Dis 2003, 3(12):757-771.

2. McBride AJ, Athanazio DA, Reis MG, Ko Al: Leptospirosis. Curr Opin Infect Dis 2005, 18(5):376-386.

3. Ristow $P$, Bourhy $P$, da Cruz McBride FW, Figueira CP, Huerre M, Ave P, Saint-Girons IS, Ko Al, Picardeau M: The OmpA-like protein Loa22 is essential for leptospiral virulence. PLoS Pathogens 2007, 3(7):e97.

4. Wu BT, Bao L, Sun Z, Li DK, Zhang Y: [The OmpA-like protein Loa22 from Leptospira interrogans serovar lai induces apoptosis in A549 via Ca2+ signal pathway]. Journal of Sichuan University Medical Science Edition 2011, 42(3):298-302

5. Zhang $Y$, Bao L, Zhu H, Huang B, Zhang H: OmpA-like protein Loa22 from Leptospira interrogans serovar Lai is cytotoxic to cultured rat renal cells and promotes inflammatory responses. Acta Biochimica et Biophysica Sinica 2010, 42(1):70-79.

6. Hoke DE, Egan S, Cullen PA, Adler B: LipL32 is an extracellular matrixinteracting protein of Leptospira spp. and Pseudoalteromonas tunicata. Infect Immun 2008, 76(5):2063-2069.

7. Murray GL, Srikram A, Hoke DE, Wunder EA Jr, Henry R, Lo M, Zhang K, Sermswan RW, Ko Al, Adler B: Major surface protein LipL32 is not required for either acute or chronic infection with Leptospira interrogans. Infect Immun 2009, 77(3):952-958.

8. Stevenson B, Choy HA, Pinne M, Rotondi ML, Miller MC, Demoll E, Kraiczy P, Cooley AE, Creamer TP, Suchard MA, et al: Leptospira interrogans 
endostatin-like outer membrane proteins bind host fibronectin, laminin and regulators of complement. PLoS One 2007, 2(11):e1188.

9. Barbosa AS, Abreu PA, Neves FO, Atzingen MV, Watanabe MM, Vieira ML, Morais ZM, Vasconcellos SA, Nascimento AL: A newly identified leptospiral adhesin mediates attachment to laminin. Infect Immun 2006, 74(11):6356-6364.

10. Yuri K, Takamoto Y, Okada M, Hiramune T, Kikuchi N, Yanagawa R: Chemotaxis of leptospires to hemoglobin in relation to virulence. Infect Immun 1993, 61(5):2270-2272.

11. Knirel YA, Moll H, Helbig JH, Zahringer U: Chemical characterization of a new 5,7-diamino-3,5,7,9-tetradeoxynonulosonic acid released by mild acid hydrolysis of the Legionella pneumophila serogroup 1 lipopolysaccharide. Carbohydr Res 1997, 304(1):77-79.

12. Neumeister B, Faigle M, Sommer M, Zahringer U, Stelter F, Menzel R, Schutt C, Northoff H: Low endotoxic potential of Legionella pneumophila lipopolysaccharide due to failure of interaction with the monocyte lipopolysaccharide receptor CD14. Infect Immun 1998, 66(9):4151-4157.

13. Goon S, Kelly JF, Logan SM, Ewing CP, Guerry P: Pseudaminic acid, the major modification on Campylobacter flagellin, is synthesized via the Cj1293 gene. Mol Microbiol 2003, 50(2):659-671.

14. Schoenhofen IC, McNally DJ, Brisson JR, Logan SM: Elucidation of the CMP-pseudaminic acid pathway in Helicobacter pylori: synthesis from UDP-N-acetylglucosamine by a single enzymatic reaction. Glycobiology 2006, 16(9):8C-14C.

15. Hopf PS, Ford RS, Zebian N, Merkx-Jacques A, Vijayakumar S, Ratnayake D, Hayworth J, Creuzenet C: Protein glycosylation in Helicobacter pylori: beyond the flagellins? PLoS One 2011, 6(9):e25722.

16. Lewis AL, Desa N, Hansen EE, Knirel YA, Gordon Jl, Gagneux P, Nizet V, Varki A: Innovations in host and microbial sialic acid biosynthesis revealed by phylogenomic prediction of nonulosonic acid structure. Proc Natl Acad Sci U S A 2009, 106(32):13552-13557.

17. Rangarajan ES, Proteau A, Cui Q, Logan SM, Potetinova Z, Whitfield D, Purisima EO, Cygler M, Matte A, Sulea T, et al: Structural and functional analysis of Campylobacter jejuni PseG: a udp-sugar hydrolase from the pseudaminic acid biosynthetic pathway. J Biol Chem 2009, 284(31):20989-21000.

18. Schoenhofen IC, Vinogradov E, Whitfield DM, Brisson JR, Logan SM: The CMP-legionaminic acid pathway in Campylobacter: biosynthesis involving novel GDP-linked precursors. Glycobiology 2009, 19(7):715-725.

19. Morrison JP, Schoenhofen IC, Tanner ME: Mechanistic studies on PseB of pseudaminic acid biosynthesis: a UDP-N-acetylglucosamine 5-inverting 4,6-dehydratase. Bioorganic Chemistry 2008, 36(6):312-320.

20. Schoenhofen IC, Lunin W, Julien JP, Li Y, Ajamian E, Matte A, Cygler M, Brisson JR, Aubry A, Logan SM, et al: Structural and functional characterization of $\mathrm{PseC}$, an aminotransferase involved in the biosynthesis of pseudaminic acid, an essential flagellar modification in Helicobacter pylori. J Biol Chem 2006, 281(13):8907-8916.

21. Schoenhofen IC, McNally DJ, Vinogradov E, Whitfield D, Young NM, Dick S, Wakarchuk WW, Brisson JR, Logan SM: Functional characterization of dehydratase/aminotransferase pairs from Helicobacter and Campylobacter: enzymes distinguishing the pseudaminic acid and bacillosamine biosynthetic pathways. J Biol Chem 2006, 281(2):723-732.

22. Hao J, Balagurumoorthy P, Sarilla S, Sundaramoorthy M: Cloning, expression, and characterization of sialic acid synthases. Biochem Biophys Res Commun 2005, 338(3):1507-1514.

23. Shang ES, Summers TA, Haake DA: Molecular cloning and sequence analysis of the gene encoding LipL41, a surface-exposed lipoprotein of pathogenic Leptospira species. Infect Immun 1996, 64(6):2322-2330.

24. Koizumi $\mathrm{N}$, Watanabe $\mathrm{H}$ : Molecular cloning and characterization of a novel leptospiral lipoprotein with OmpA domain. FEMS Microbiol Lett 2003, 226(2):215-219.

25. Cullen PA, Xu X, Matsunaga J, Sanchez Y, Ko Al, Haake DA, Adler B: Surfaceome of Leptospira spp. Infect Immun 2005, 73(8):4853-4863.

26. Nally JE, Whitelegge JP, Aguilera R, Pereira MM, Blanco DR, Lovett MA: Purification and proteomic analysis of outer membrane vesicles from a clinical isolate of Leptospira interrogans serovar Copenhageni. Proteomics 2005, 5(1):144-152

27. Nally JE, Whitelegge JP, Bassilian S, Blanco DR, Lovett MA: Characterization of the Outer Membrane Proteome of Leptospira interrogans Expressed during Acute Lethal Infection. Infect Immun 2007, 75(2):766-773.
28. Malmstrom J, Beck M, Schmidt A, Lange V, Deutsch EW, Aebersold R: Proteome-wide cellular protein concentrations of the human pathogen Leptospira interrogans. Nature 2009, 460(7256):762-765

29. Jurcisek J, Greiner L, Watanabe H, Zaleski A, Apicella MA, Bakaletz LO: Role of sialic acid and complex carbohydrate biosynthesis in biofilm formation by nontypeable Haemophilus influenzae in the chinchilla middle ear. Infect Immun 2005, 73(6):3210-3218.

30. Carlin AF, Lewis AL, Varki A, Nizet V: Group B streptococcal capsular sialic acids interact with siglecs (immunoglobulin-like lectins) on human leukocytes. J Bacteriol 2007, 189(4):1231-1237.

31. Carlin AF, Uchiyama S, Chang YC, Lewis AL, Nizet V, Varki A: Molecular mimicry of host sialylated glycans allows a bacterial pathogen to engage neutrophil Siglec- 9 and dampen the innate immune response. Blood 2009, 113(14):3333-3336.

32. Jones C, Virji M, Crocker PR: Recognition of sialylated meningococcal lipopolysaccharide by siglecs expressed on myeloid cells leads to enhanced bacterial uptake. Mol Microbiol 2003, 49(5):1213-1225.

33. Asakura H, Churin Y, Bauer B, Boettcher JP, Bartfeld S, Hashii N, Kawasaki N, Mollenkopf HJ, Jungblut PR, Brinkmann V, et al: Helicobacter pylori HP0518 affects flagellin glycosylation to alter bacterial motility. Mol Microbiol 2010, 78(5):1130-1144.

34. van Alphen LB, Wuhrer M, Bleumink-Pluym NM, Hensbergen PJ, Deelder $A M$, van Putten JP: A functional Campylobacter jejuni maf4 gene results in novel glycoforms on flagellin and altered autoagglutination behaviour. Microbiology 2008, 154(Pt 11):3385-3397.

35. Schirm M, Soo EC, Aubry AJ, Austin J, Thibault P, Logan SM: Structural, genetic and functional characterization of the flagellin glycosylation process in Helicobacter pylori. Mol Microbiol 2003, 48(6):1579-1592.

36. Guerry $P$, Ewing CP, Schirm M, Lorenzo M, Kelly J, Pattarini D, Majam $G$, Thibault $P$, Logan $S$ : Changes in flagellin glycosylation affect Campylobacter autoagglutination and virulence. Mol Microbiol 2006 60(2):299-311.

37. Post DM, Yu L, Krasity BC, Choudhury B, Mandel MJ, Brennan CA, Ruby EG, McFall-Ngai MJ, Gibson BW, Apicella MA: The O-antigen and core carbohydrate of Vibrio fischeri lipopolysaccharide: Composition and analysis of their role in Euprymna scolopes light organ colonization. J Biol Chem 2012, 287(11):8515-8530.

38. Bey RF, Johnson RC: Protein-free and low-protein media for the cultivation of Leptospira. Infect Immun 1978, 19(2):562-569.

39. Lewis AL, Lubin JB, Argade S, Naidu N, Choudhury B, Boyd EF: Genomic and metabolic profiling of nonulosonic acids in Vibrionaceae reveal biochemical phenotypes of allelic divergence in Vibrio vulnificus. Appl Environ Microbiol 2011, 77(16):5782-5793.

40. Lewis AL, Nizet $V$, Varki A: Discovery and characterization of sialic acid O-acetylation in group B Streptococcus. Proc Natl Acad Sci U S A 2004, 101(30):11123-11128.

41. Klein A, Diaz S, Ferreira I, Lamblin G, Roussel P, Manzi AE: New sialic acids from biological sources identified by a comprehensive and sensitive approach: liquid chromatography-electrospray ionization-mass spectrometry (LC-ESIMS) of SIA quinoxalinones. Glycobiology 1997, 7(3):421-432.

doi:10.1186/1471-2180-12-161

Cite this article as: Ricaldi et al.: Expression of sialic acids and other nonulosonic acids in Leptospira. BMC Microbiology 2012 12:161.

\section{Submit your next manuscript to BioMed Central and take full advantage of:}

- Convenient online submission

- Thorough peer review

- No space constraints or color figure charges

- Immediate publication on acceptance

- Inclusion in PubMed, CAS, Scopus and Google Scholar

- Research which is freely available for redistribution 